Seit Inkrafttreten des AGG ist das juristische Verstehen zum Thema der Entgeltgleichheit bei gleichwertiger Arbeit nicht beträchtlich größer geworden. Das AGG hat für diesen Rechtsanspruch, der unionsrechtlich nach wie vor unverändert besteht und damit auch auf nationaler Ebene rechtlich durchgesetzt werden kann, eher eine Verschlechterung gebracht: Im AGG ist dieser Rechtsanspruch nicht ausdrücklich formuliert und damit relativ „unsichtbar“. Der frühere $\$ 612$ Absatz 3 BGB ist seitdem nicht mehr in Kraft. Damit ist die ehemals klare Aussage „darf für gleiche oder gleichwertige Arbeit nicht wegen des Geschlechts des Arbeitnehmers eine geringere Vergütung vereinbart werden als bei einem Arbeitnehmer des anderen Geschlechts“ aus dem BGB verschwunden, jedoch im AGG nicht vergleichbar ausdrücklich formuliert worden. Es verwundert kaum, dass dementsprechend der kommentierende Widerhall dieses Rechtsanspruchs in den einschlägigen Kommentaren zum AGG relativ dürftig ist. Dies beginnt schon damit, dass dieses Thema, da im AGG kein Paragraph ausdrücklich davon handelt, auch in den Kommentierungen keinen „Ort“ hat, an dem es einheitlich zu finden wäre.

\section{Quo vadis?}

Der Stand der Dinge ist heute - mehr als fünfzig Jahre nach Unterzeichnung der Römischen Verträge - in Deutschland also bisher keine Erfolgsgeschichte. Gibt es derzeit Grund zu der Annahme, dass sich daran etwas ändert? Jedenfalls ist einiges an Bewegung und Diskussion bis hin zu Gesetzgebungsinitiativen zu verzeichnen, damit aus dem Rechtsanspruch auf Entgeltgleichheit Rechtswirklichkeit wird. ${ }^{14}$

Arbeit nicht abhängig vom Geschlecht oder einem anderen verbotenen Differenzierungsgrund wie Alter, Behinderung oder Herkunft zu bewerten, ist ein Gebot der Fairness. All diese Gebote der Fairness sind als Diskriminierungsverbote geltendes Recht. Ein Mehr an inter-/transdisziplinärer Zusammenarbeit - angefangen beispielsweise bei Schulungsveranstaltungen - kann aus meiner Sicht einen Beitrag dazu leisten, daraus Rechtswirklichkeit werden zu lassen.

14 Vgl. dazu insbesondere Pfarr (2011, Fn. 3) sowie Pfarr und weitere Beiträge in dieser djbz.

\title{
Aktuelle Entwicklungen auf EU-Ebene zur Bekämpfung von Entgeltdiskriminierung
}

\section{(Auswahl - Stand Juni 2012)}

Entschließung des Europäischen Parlaments vom 24. Mai 2012 mit Empfehlungen an die Kommission zur Anwendung des Grundsatzes des gleichen Entgelts für Männer und Frauen bei gleicher oder gleichwertiger Arbeit (2011/2285(INI)).

- Daraus (Auszug unter Auslassung einzelner Punkte):

Das Europäische Parlament

- fordert die Kommission auf, bis spätestens 15. Februar 2013 die Richtlinie 2006/54/EG zu überprüfen und Änderungen im Sinne von Artikel 157 AEUV unter Beachtung der dieser Entschließung beigefügten ausführlichen Empfehlungen zumindest in Bezug auf die folgenden Aspekte des geschlechtsbezogenen Lohngefälles vorzuschlagen:

- Begriffsbestimmungen,

- Analyse der Situation und Transparenz der Ergebnisse,

- Arbeitsplatzbewertung und berufliche Einstufung,

- Gleichstellungsgremien und Rechtsmittel,

- sozialer Dialog,

- Verhütung von Diskriminierung,

- Gender Mainstreaming,

- Sanktionen,

- Straffung der Unionsregelungen und -politik.

- ist sich darüber im Klaren, dass es vielfältige Gründe für eine Verschärfung des Lohngefälles gibt, und stellt deshalb fest, dass ein Ansatz, der mehrere Ebenen und mehrere Facetten einbezieht, der Union eine starke Führungsrolle bei der Koordination der Maßnahmen, der Förderung bewährter Methoden und dem Einbinden verschiedener Handlungsträger, zum Beispiel der europäischen Sozialpartner und nichtstaatlicher Organisationen, abverlangt und das Ziel verfolgt, eine euro- paweite Strategie zur Bekämpfung des geschlechtsspezifischen Lohngefälles zu entwickeln;

- fordert die Mitgliedstaaten auf, die neu gefasste Richtlinie 2006/54/EG einheitlich umzusetzen und durchzusetzen, den Privatsektor wie auch den öffentlichen Sektor anzuregen, eine aktivere Rolle beim Abbau des geschlechtsspezifischen Lohngefälles zu spielen; ist der Ansicht, dass die Mitgliedstaaten und die Kommission die Sozialpartner einschließlich der Arbeitgeber auffordern sollten, Arbeitsbewertungssysteme zu schaffen, die geschlechtsneutral sind, Systeme der beruflichen Einstufung umzusetzen und das Konzept des Arbeitsplatzes mit gleichem Entgelt zu unterstützen;

- fordert die Mitgliedstaaten auf, bei der Bekämpfung der Unterschiede im Bereich des Entgelts, von denen Frauen in den öffentlichen Verwaltungen, Einrichtungen und Unternehmen generell betroffen sind, mit gutem Beispiel voranzugehen;

- betont, wie wichtig Tarifverhandlungen und Tarifverträge beim Kampf gegen die Diskriminierung von Frauen sind, insbesondere in den Bereichen Zugang zur Beschäftigung, Löhne, Arbeitsbedingungen, beruflicher Aufstieg und Berufsbildung;

- begrüßt die Initiative der Kommission zur Einführung eines Europäischen Tags der Lohngleichheit, der erstmals am 5. März 2011 stattfand und zum zweiten Mal am 2. März 2012 begangen wurde;

- stellt fest, dass Lohnunterschiede, die auf andere Faktoren wie Rasse, ethnische Zugehörigkeit, sexuelle Ausrichtung oder Religion zurückzuführen sind, nicht hingenommen werden dürfen; 
- ermutigt die Kommission, eine engere Koordinierung unter den Mitgliedstaaten hinsichtlich der Forschung, Analyse und der bestmöglichen Nutzung der Weitergabe von bewährten Methoden zu stärken;

- fordert die Mitgliedstaaten auf, bewährte Verfahren auszutauschen und die Zusammenarbeit bei der Entwicklung neuer Konzepte zur Bekämpfung des Lohngefälles zwischen den Geschlechtern zu intensivieren, und zwar nach Möglichkeit unter Einbindung der Sozialpartner;

- fordert die Kommission und die Mitgliedstaaten auf, den Gehaltsunterschieden zwischen den Geschlechtern im Rahmen aller relevanten unionsweiten politischen Maßnahmen und einzelstaatlichen Programme, insbesondere der auf Armutsbekämpfung ausgerichteten, entgegenzutreten;

- regt an, dass die Mitgliedstaaten eine/n Beauftragte/n für gleiches Entgelt ernennen, der/die die Situation in den einzelnen Mitgliedstaaten verfolgt und den nationalen Parlamenten wie auch dem Europäischen Parlament über die erzielten Fortschritte berichtet;

- fordert die Sozialpartner auf, die Verantwortung für die Schaffung einer gleichberechtigteren Lohnstruktur für beide Geschlechter auf sich zu nehmen, Kurse für Verhandlungskompetenzen, u.a. im Bereich der Lohn- und Gehaltsverhandlungen, anzubieten, ferner in einem ersten Schritt das Bewusstsein für gleiches Entgelt zu stärken, wobei längerfristig ,Lohn-Audits“ vorgeschrieben werden sollen, und die Stellung der Frau in der Struktur der Sozialpartnerschaft, insbesondere in Führungspositionen, zu stärken;

- fordert die Mitgliedstaaten auf, die Möglichkeit einer Sammelklage wegen Verstößen gegen den Grundsatz des gleichen Entgelts vorzusehen als ein Instrument, in dessen Rahmen Einzelpersonen und/oder Vertreterorganisationen einen Fall im Namen von Beschwerdeführern, die darin einwilligen, vor Gericht bringen können und NRO und Gewerkschaften Klagebefugnis erhalten, so dass sie Opfer von Diskriminierung auch in Verwaltungsverfahren vertreten können; fordert die Kommission auf, die Aufnahme von Sammelklagen bei Verstößen gegen den Grundsatz des gleichen Entgelts in ihren künftigen Vorschlag für eine horizontale Richtlinie über Sammelklagen zu prüfen;

- unterstreicht, dass nur sehr wenige Klagen betreffend die Lohndiskriminierung aus Gründen des Geschlechts vor ein zuständiges (ordentliches oder Verwaltungs-) Gericht gebracht wurden; fordert die Kommission und die Mitgliedstaaten daher auf, ihre Sensibilisierungskampagnen fortzusetzen, wozu auch angemessene Informationen über die Beweislast gehören, da diese erheblich dazu beiträgt, den Grundsatz des gleichen Entgelts zu gewährleisten;

vertritt die Auffassung, dass die Verfahren und Mechanismen zur Wahrung des Grundsatzes des gleichen Entgelts für gleichwertige Arbeit sowie zum Verbot jeglicher Diskriminierung aus Gründen des Geschlechts verbessert und vereinfacht werden müssen;
- ruft die Mitgliedstaaten sowie die Verbände der Arbeitnehmer und Arbeitgeber auf, gemeinsam objektive Arbeitsplatzbewertungsinstrumente zu entwickeln, um das Lohngefälle zwischen Männern und Frauen zu verringern;

- fordert die Mitgliedstaaten dazu auf, Ziele, Strategien und Fristen für den Abbau des geschlechtsspezifischen Lohngefälles und die Verwirklichung des gleichen Entgelts für gleiche oder gleichwertige Arbeit festzulegen.

- Federführend war im Vorfeld der Ausschuss für die Rechte der Frau und die Gleichstellung der Geschlechter (FEMM).

- Link/Fundstelle: http://www.europarl.europa.eu/sides/ getDoc.do?pubRef=-//EP//TEXT+TA+P7-TA-2012-0225+O+ $\mathrm{DOC}+\mathrm{XML}+\mathrm{Vo} / / \mathrm{DE}$

Mitteilung der Kommission vom 21. September 2010 „Strategie für die Gleichstellung von Frauen und Männern 20102015" (KOM(2010)0491)

- Daraus: „Leitaktionen“- Die Kommission wird

- gemeinsam mit den Sozialpartnern auf europäischer Ebene und unter Wahrung der Autonomie des sozialen Dialogs untersuchen, wie die Lohntransparenz verbessert werden kann und welche Auswirkungen Vertragsformen wie Teilzeit oder befristete Arbeitsverträge auf die Lohngleichheit haben.

- Initiativen für gleiches Entgelt am Arbeitsplatz - wie etwa Garantiesiegel, Selbstverpflichtungen und Auszeichnungen für vorbildliche Arbeitgeber/innen sowie die Entwicklung von Instrumenten unterstützen, die es Arbeitgebern erlauben, ungerechtfertigte geschlechtsspezifische Lohnunterschiede zu beseitigen.

- einen Europäischen Tag für gleiches Entgelt einführen, der jedes Jahr stattfinden und das Bewusstsein dafür schärfen soll, wie viele Stunden mehr Frauen arbeiten müssen, um so viel zu verdienen wie Männer.

- Frauen dazu ermutigen, sich für nichttraditionelle Berufe zu entscheiden, $z$. B. in der Umweltbranche oder anderen innovativen Sektoren.

- Link/Fundstelle: http://eur-lex.europa.eu/LexUriServ/ LexUriServ.do?uri=COM:2010:0491:FIN:DE:PDF

Bericht der Europäischen Kommission vom Mai 2010 mit dem Titel „Der geschlechtsspezifische Verdienstabstand in Europa aus rechtlicher Sicht" (Verfasserin: Petra Foubert)

- Link/Fundstelle: http://ec.europa.eu/justice/

gender-equality/files/gender_pay_gap/

genderpaygapfromlegalperspective-nov2010_de.pdf

Mitteilung der Kommission vom 5. März 2010 „Ein verstärktes Engagement für die Gleichstellung von Frauen und Männern - eine Frauen-Charta“ (KOM(2010)0078)

- Daraus: „Wir bekräftigen unser Engagement für eine entschlossene Mobilisierung sämtlicher Instrumente, sowohl legislativer als auch nichtlegislativer Art, zum Abbau des geschlechtsspezifischen Lohngefälles. Die ungleiche Bezahlung von Frauen und Männern verursacht Kosten, die sich Europa nicht leisten kann. Gemeinsam mit den 27 Mitgliedstaaten der EU werden wir darauf hinarbeiten, das 
geschlechtsspezifische Lohngefälle in der Europäischen Union bis zum Ende der Amtszeit dieser Europäischen Kommission deutlich zu verringern."

- Link/Fundstelle: http://eur-lex.europa.eu/LexUriServ/ LexUriServ.do?uri=CELEX:52010DCoo78:de:NOT

Unionsrecht: Richtlinie 2006/54/EG des Europäischen Parlaments und des Rates vom. 5. Juli 2006 zur Verwirklichung des Grundsatzes der Chancengleichheit und Gleichbehandlung von Männern und Frauen in Arbeits- und Beschäftigungsfragen

- Daraus: Artikel 4-Gleiches Entgelt/Diskriminierungsverbot

- Bei gleicher Arbeit oder bei einer Arbeit, die als gleichwertig anerkannt wird, wird mittelbare und unmittel- bare Diskriminierung aufgrund des Geschlechts in Bezug auf sämtliche Entgeltbestandteile und -bedingungen beseitigt.

- Insbesondere wenn zur Festlegung des Entgelts ein System beruflicher Einstufung verwendet wird, muss dieses System auf für männliche und weibliche Arbeitnehmer gemeinsamen Kriterien beruhen und so beschaffen sein, dass Diskriminierungen aufgrund des Geschlechts ausgeschlossen werden.

- Link/Fundstelle: http://eur-lex.europa.eu/LexUriServ/ LexUriServ.do?uri=OJ:L:2006:204:0023:0036:de:PDF

\section{Diskriminierungsfreie Beurteilung von Leistung, Eignung und Befähigung?}

\author{
Dr. Karin Tondorf \\ Mitglied der Kommission Arbeits-, Gleichstellungs- und Wirt- \\ schaftsrecht des djb; Freie Wissenschaftlerin/Beraterin, Seddiner \\ See
}

\section{Dr. Andrea Jochmann-Döll}

Freie Wissenschaftlerin/Beraterin, GEFA Forschung + Beratung, Essen

In Deutschland erhalten Frauen noch immer ein wesentlich geringeres Arbeitsentgelt als Männer: der Verdienstunterschied verharrt seit mehreren Jahren bei 23 Prozent. Eine Ursache für diese Entgeltlücke wird darin gesehen, dass Frauen vielfach Tätigkeiten mit geringeren Anforderungen verrichten als Männer oft genug unfreiwillig. Denn viele Frauen sind motiviert und hinsichtlich ihrer Qualifikation auch in der Lage, höherwertige Tätigkeiten zu übernehmen. Jedoch fällt die Auswahlentscheidung bei der Stellenbesetzung oder Beförderung nicht immer zu ihren Gunsten aus. Da eine ablehnende Entscheidung für die nachteilig Betroffenen oft schwer durchschaubar und kaum nachvollziehbar ist, kommen mitunter Zweifel auf, ob allein sachliche Gründe ausschlaggebend waren oder ob eine Benachteiligung aufgrund des Geschlechts vorliegt. Damit geraten Beurteilungen in den Blick, die, vor allem im öffentlichen Dienst und für die dort beschäftigten Beamten und Beamtinnen, eine wesentliche Grundlage für Stellenbesetzungen und Beförderungen darstellen. Die Vermutung, Beurteilungen könnten ein nicht unerhebliches Diskriminierungspotenzial beinhalten, ist weder neu noch „aus der Luft gegriffen“. Anhaltspunkte hierfür liefern wissenschaftliche Untersuchungen ${ }^{1}$, Praxiserfahrungen ${ }^{2}$ und vereinzelt bereits veröffentlichte Beurteilungsstatistiken für den öffentlichen Dienst. So erhielten zum Beispiel nur 25,5 Prozent der beurteilten Frauen, jedoch 75 Prozent der beurteilten Männer einer Landesverwaltung die beiden höchsten Punktzahlen. ${ }^{3}$
Im Mittelpunkt dieses Beitrags steht die Frage, mit welchen Instrumenten die Gleichbehandlung der Geschlechter bei der Beurteilung geprüft werden kann. Es werden zwei Prüfinstrumente vorgestellt, die auf gleichstellungsrechtlichen Maßstäben basieren und von interessierten Praktiker/inne/n (Arbeitgeber/ inne/n, Gleichstellungsbeauftragten, Personalrät/inn/en) und Rechtsexpert/inn/en angewendet werden können. Diese Instrumente wurden kürzlich von den Autorinnen in einem wissenschaftlichen Projekt angewendet, bei dem es um die Analyse der Gleichbehandlung der Geschlechter bei der Beurteilung im Polizeivollzugsdienst der Bundesländer und der Bundespolizei geht. ${ }^{4}$ (s. auch Informationen im Kasten)

1 Dudek, Sonja M., Diversity in Uniform. Geschlecht und Migrationshintergrund in der Berliner Schutzpolizei, Wiesbaden 2009; Fried, Andrea/Wetzel, Ralf/Baitsch, Christof, Wenn zwei das Gleiche tun.... Diskriminierungsfreie Personalbeurteilung, Zürich 2000; Krell, Gertraude, „Diskriminierungs- und Gleichstellungspotenzial von Leistungsbeurteilungen“, in: Krell, Gertraude/Ortlieb, Renate/Sieben, Barbara (Hg.), Chancengleichheit durch Personalpolitik, Wiesbaden 2011, S. 261-274; Tondorf, Karin/Jochmann-Döll, Andrea, (Geschlechter-)Gerechte Leistungsvergütung? Vom (Durch-)Bruch des Leistungsprinzips in der Entlohnung, Hamburg 2005.

2 Schreyögg, Friedel, Praxisbeispiel München: „Beurteilungsverfahren sind nicht geschlechtsneutral“, in: Krell, Gertraude/Ortlieb, Renate/Sieben, Barbara (Hg.), Chancengleichheit durch Personalpolitik, Wiesbaden 2011, S. 275-282; Niedersächsisches Ministerium für Soziales, Frauen, Familie und Gesundheit (Hg.): GemiNi - Gender Mainstreaming in Niedersachsen, Grundlagen und Beispiele, Hannover 2005

3 Tondorf, Karin/Jochmann-Döll, Andrea, (Geschlechter-)Gerechte Leistungsvergütung? Vom (Durch-)Bruch des Leistungsprinzips in der Entlohnung, Hamburg 2005, S. 88; vgl. für den Polizeivollzugsdienst: Auswertung der Beurteilungsergebnisse, in: Streife 10/2000, S. 18 f. sowie Dienstliche Beurteilung der Polizeikräfte in Bayern im Jahr 2009 und Chancengleichheit in Teilzeitarbeit, Schriftliche Anfrage an den Bayerischen Landtag, Landtagsdrucksache 16/3359 v. 2.3.2010.

4 Jochmann-Döll, Andrea/Tondorf, Karin, Beurteilungen im Polizeivollzugsdienst - Gewährleistung der Gleichstellung der Geschlechter?, gefördert von der Hans-Böckler-Stiftung, Projektnr. S-2012520-3, noch nicht abgeschlossen und veröffentlicht. 